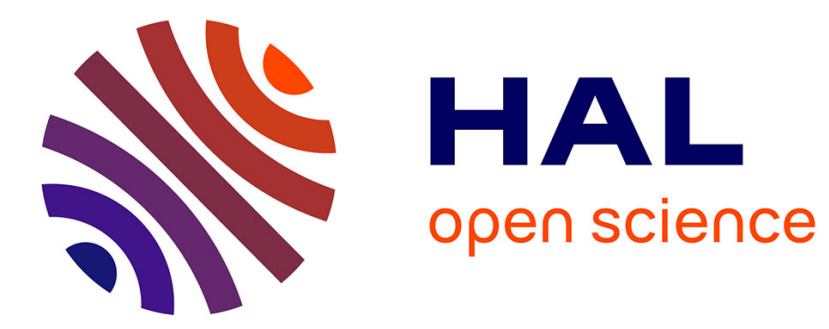

\title{
Stability Analysis of a New Extended L1 Controller with Experimental Validation on an Underwater Vehicle
}

\author{
Divine Maalouf, Ahmed Chemori, Vincent Creuze
}

\section{To cite this version:}

Divine Maalouf, Ahmed Chemori, Vincent Creuze. Stability Analysis of a New Extended L1 Controller with Experimental Validation on an Underwater Vehicle. CDC: Conference on Decision and Control, Dec 2013, Florence, Italy. lirmm-00847170

\section{HAL Id: lirmm-00847170 \\ https://hal-lirmm.ccsd.cnrs.fr/lirmm-00847170}

Submitted on 22 Jul 2013

HAL is a multi-disciplinary open access archive for the deposit and dissemination of scientific research documents, whether they are published or not. The documents may come from teaching and research institutions in France or abroad, or from public or private research centers.
L'archive ouverte pluridisciplinaire HAL, est destinée au dépôt et à la diffusion de documents scientifiques de niveau recherche, publiés ou non, émanant des établissements d'enseignement et de recherche français ou étrangers, des laboratoires publics ou privés. 


\title{
Stability Analysis of a New Extended $\mathcal{L}_{1}$ Controller with Experimental Validation on an Underwater Vehicle
}

\author{
Divine Maalouf, Ahmed Chemori, and Vincent Creuze
}

\begin{abstract}
L}_{1}$ adaptive control is a recent scheme elaborated in order to ensure a decoupling between robustness and fast adaptation. This controller was validated in simulations and experimental results. Research is currently being developped in order to improve its architecture and design challenges. Our study focuses on the inherent time lag observed in presence of a varying reference trajectory. The solution proposed in this regard is the extended $\mathcal{L}_{1}$ controller in which we suggest an augmentation of the original one with a PID aiming to reduce the tracking error. The stability analysis of this new scheme is shown in this paper. Experimental results on an underwater vehicle subjected to a varying trajectory under several disturbances are then displayed to illustrate the efficiency of the method.
\end{abstract}

\section{INTRODUCTION}

With the advances of robotics and the industrial growth, various challenges in nonlinear control saw the light. Among the popular schemes dealing with varying systems and robustness, adaptive control was born in order to provide an online estimation of unknown or varying model parameters. Many methods were elaborated under this family with the aim of improving it in order to yield a better performance. In fact, such controllers are known to exhibit bad transition behaviors during the adaptation of the parameters. This leads to the compromise between a slower adaptation (use of small gains) and a fast adaptation (use of larger gains) with the loss of robustness. Initial parameter intialization is also often required in order to facilitate the parameter convergence. A study was made in [1] highlighting the problems encountered in adaptive control. The recently developped $\mathcal{L}_{1}$ adaptive controller [2] stands out among all other developped adaptive methods in its particular architecture where robustness and adaptation are decoupled. In fact the low pass filter introduced in its structure separates the estimation loop from the control loop. The result is therefore a fast adaptation with the ability of using very large gains without harming the robustness of the closed-loop system. With such a novel method, various previously noted failures in adaptive control were revisited [3] [4]. Given that this controller is still recent, many improvements and new variations in its implementation were proposed in litterature. We find for instance methods for estimating the parameters in the adaptation part such as a straightforward proportional adaptation law [5], a switching law with a dead zone [6], or an adaptive observer with a projection operator [7]. We also find a systematic way

The authors would like to thank the Tecnalia foundation for its support. Divine Maalouf, Ahmed Chemori and Vincent Creuze are with the LIRMM, Université Montpellier 2 (UM2)-CNRS, 161 rue Ada, 34392 Montpellier, France, ahmed.chemori@lirmm. fr for designing the filter in order to achieve the desired performance [8]. In this paper, we propose an extension of the $\mathcal{L}_{1}$ controller with a proportional integral derivative (PID) controller augmentation in order to solve the time lag observed when this scheme is implemented on systems with a varying trajectory. In fact, similarly to Model Reference Adaptive Control (MRAC), only the boundedness of the error is guaranteed for time varying reference trajectories thus leading to time lags. Indeed, asymptotic convergence is proven only when the reference trajectory is constant. Moreover, the presence of the filter in the control loop increases the observed time lag. Augmenting the original $\mathcal{L}_{1}$ controller with a proportional integral derivative controller improves the tracking performance without harming the robustness of the system. We have recently experimentally shown that a PI augmentation of the $\mathcal{L}_{1}$ controller improves trajectory following by drastically decreasing the time lags [9]. The robustness towards a parameter variation was also validated through a scenario where the robot's buoyancy was modified. The scope of this paper is not only to extend the proposed augmentation to the PID case, but also to give an analysis of the stability of the PID augmented $\mathcal{L}_{1}$ controller. For this purpose, we use methods based on the Nyquist criterion and on stability margins. An example is given to show that the system remains stable despite the added PID extension. Moreover, experimental results are provided on an underwater vehicle in depth. The robot is expected to follow a varying reference trajectory and the robustness is put at stake through several punctual disturbances. This paper is organized as follows: in the next section we describe the extended $\mathcal{L}_{1}$ adaptive control architecture, the third section gives an analysis of the closed-loop stability of the original and the extended controller when a simple linear system is given. The fourth section explains the application of this controller on the studied underwater vehicle and in the fifth section the obtained experimental results are displayed. Finally the paper ends with some concluding remarks.

\section{EXTENDED $\mathcal{L}_{1}$ ARCHITECTURE}

The establishment of the framework of the $\mathcal{L}_{1}$ adaptive controller lies in the decoupling between the adaptation and the robustness [2]. For that, the architecture of this scheme is constructed of 3 main parts as shown in Fig. 1: the state predictor, the adaptation phase and the control law formulated with a low pass filter. The estimation loop being decoupled from the the control loop garantees a fast adaptation of the studied system in presence of very large gains while preserving a good transition performance. The 


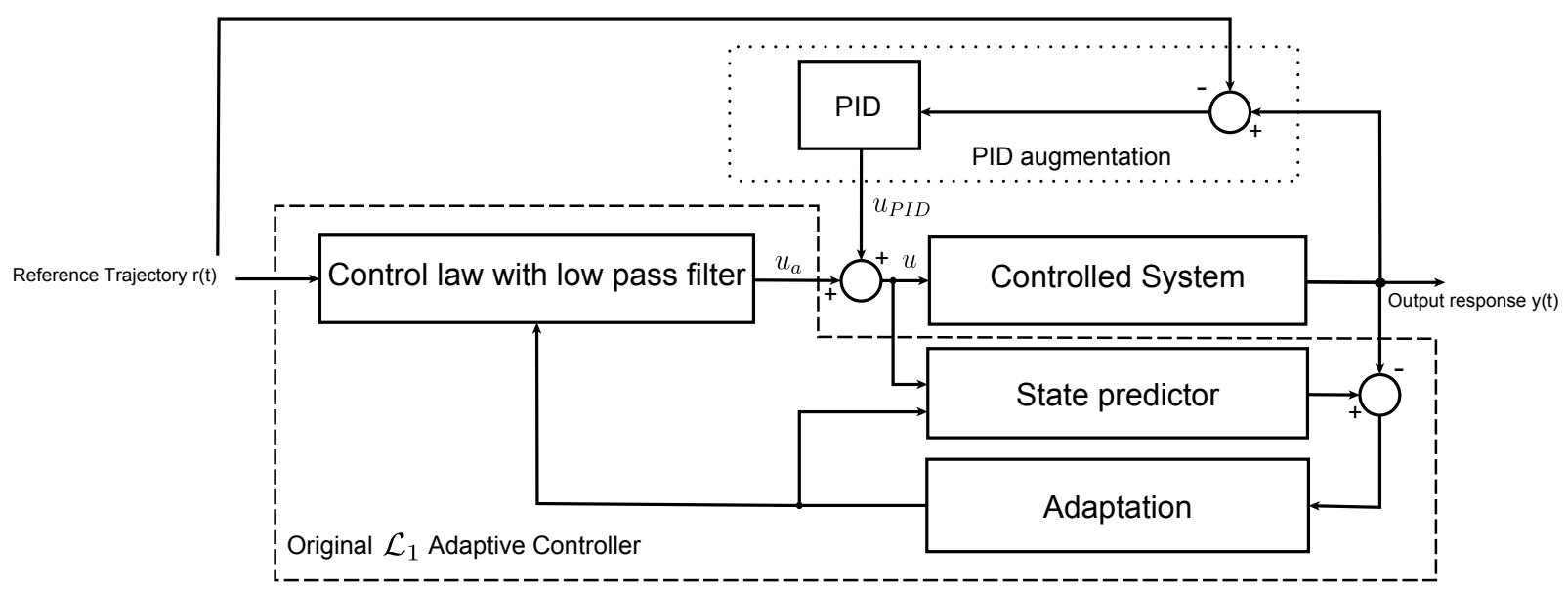

Fig. 1: Block diagram of the $\mathcal{L}_{1}$ adaptive controller

error between the measured states of the system and the estimated ones will be used for the adaptation process that is made in presence of very large gains. The resulting estimated parameters will then be used for the calculation of the control input that is filtered before being sent to the controlled system and to the state predictor. In presence of a varying trajectory, asymptotic stability is not garanteed but similarly to other adaptive schemes (for example MRAC), the tracking error is bounded. Nevertheless, in this case, the filter of the $\mathcal{L}_{1}$ adaptive controller incorporates an additional time lag of the system response with respect to the desired trajectory. The actual solution to overcome this problem is a careful design of this filter in order to ensure the adequate trade off between closed-loop desired performances and robustness. To overcome the need of a fine design and tuning of the low-pass filter, we propose in this paper to extend the $\mathcal{L}_{1}$ controller by augmenting it with a PID controller as shown in dotted lines in Fig. 1. The filtered control input $u_{a}$ incorporating the adaptation of the unknown parameters is summed to a new input $u_{P I D}$ to get the final input $u=u_{a}+u_{P I D}$ that is sent to the controlled system as well as to the state predictor. The improvement brought to the performance of the controller is therefore the reduction of the tracking error in presence of a varying reference trajectory. Experimental results are displayed in section V (cf. Fig. 7) for an underwater vehicle moving in depth comparing the performance of the original and extended $\mathcal{L}_{1}$ controller in terms of trajectory following. The robustness of this new architecture will be validated in the next section on an example given in [2] using tools from linear control showing that the robustness is still preserved for a wide range of controlled gains.

\section{CLOSED-LOOP STABILITY ANALYSIS}

In this section, we use methods known from linear control to prove the robustness of the extended architecture: the Nyquist criterion and the stability margins. For this scope, a linear system is used (cf. Fig. 2 for which the open loop transfer function is computed). This linearization is valid as long as the projection included in the adaptation stage of the $\mathcal{L}_{1}$ controller is not required (i.e no saturation of the estimated parameters). This reasonable assumption is valid with the simulation example taken and tested for different added disturbances. Consequently, the Nyquist plot is diplayed and the stability margins (phase and gain margins) are given for various design parameters. In our case, the gains of the PID and the adaptation gain will be changed in order to observe their effects on the robustness of the closed loop system.

\section{A. Illustrative example for the proof of stability}

The illustrative example using the extended $\mathcal{L}_{1}$ controller and shown in Fig. 2 can be explicitly written according to the following:

Controlled system:

$$
\dot{x}(t)=-x(t)+\theta(t)+u(t)
$$

with $x(t)$ being the state of the system, $\theta$ the unknown disturbance and $u(t)$ the control input.

State predictor:

$$
\dot{\hat{x}}(t)=-\hat{x}(t)+\hat{\theta}(t)+u(t)
$$

where the hat symbol refers to the predicted state and estimated parameter.

Adaptation stage:

$$
\dot{\hat{\theta}}(t)=-\Gamma \tilde{x}(t)
$$

with $\tilde{x}=\hat{x}-x$ and $\Gamma$ is the adaptation gain. Given that this example deals with a linear system. The adaptation law is taken to be proportional (i.e the error is multiplied directly by the adaptation gain). Usually when implementing the $\mathcal{L}_{1}$ controller a projection operator is used in the adaptation phase in order to ensure the boundedness of the estimated parameters. 


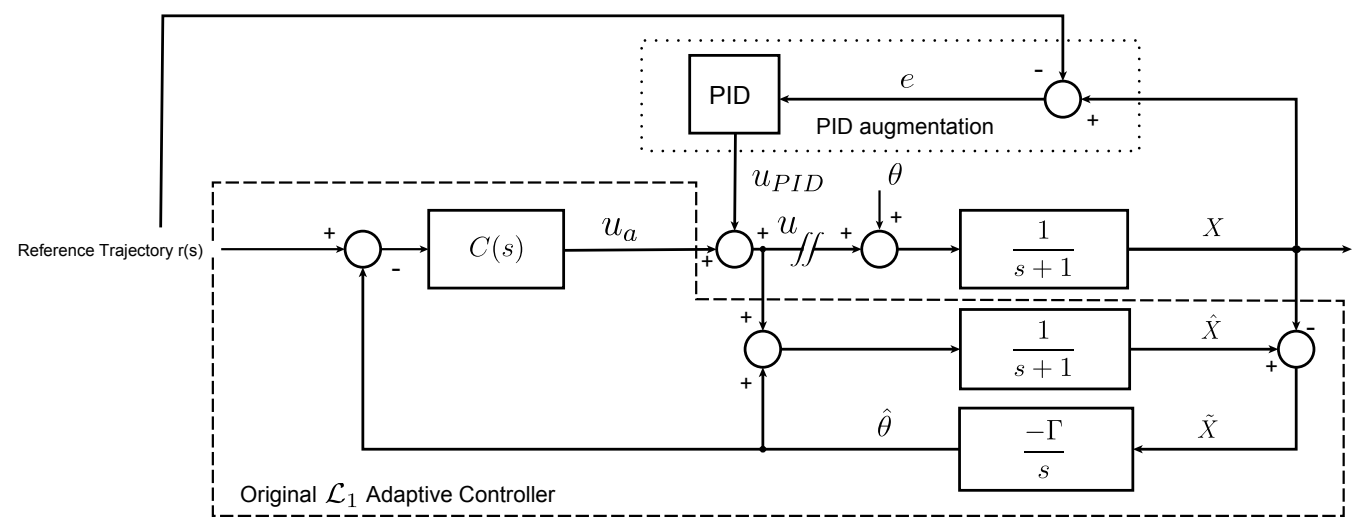

Fig. 2: Closed-loop system with the proposed extended $\mathcal{L}_{1}$ adaptive controller for the linear system developped in section III.A

\section{Control input formulation:}

$$
u(t)=-C(s)(\hat{\theta}-r(t))+u_{P I D}
$$

with $u_{P I D}=-K_{P} e(t)-K_{I} \int_{0}^{t} e(t) d t-K_{D} \frac{d e(t)}{d t}$

and $e(t)$ the tracking error defined by: $e(t)=x(t)-r(t)$ with $r(t)$ the reference trajectory.

\section{B. Comparison between the original and the extended $\mathcal{L}_{1}$ adaptive controller}

The open-loop transfer function is computed in order to calculate the stability margins of the augmented system. We break the block diagram in Fig. 2 at the level of the control input to the plant ( $\iint$ symbol). We therefore get the equation of the open-loop transfer function $G_{\text {extended }}$, with negative feedback to be:

$$
G_{\text {extended }}(s)=\frac{-\left(s+\frac{\Gamma}{s+1}\right) u_{P I D}+\Gamma C(s)}{s(s+1)+\Gamma(1-C(s))}
$$

From the above open-loop, we easily deduce the one of the nominal controller without the extension by setting $u_{p}$ to 0 and we get:

$$
G_{\text {nominal }}(s)=\frac{\Gamma C(s)}{s(s+1)+\Gamma(1-C(s))}
$$

The Nyquist plot of both open loops is shown in Fig. 3 and the stability margins in the table below for the following design parameters: $\Gamma=100000, C(s)=\frac{1}{s+1}$. The PID parameters were set to: $K_{P}=3, K_{I}=0.5$, and $K_{D}=0.2$.

Both Nyquist diagrams never encircle the critical point $(-1+j 0)$. Since the number of anti-clockwise encirclements is equal to the number of unstable poles of the open-loop transfer function, we deduce that both closed loop systems are stable. The stability margins are slightly increased for the extended controller as seen in Table I. It has to be reminded that the desired effect of the PID extension is to decrease the timelags induced in presence of a varying reference trajectory without affecting the overall closed-loop stability of the system. Then it is desired that the stability margins are kept unchanged or slightly improved.
TABLE I: Comparison of the stability margins for both controllers

\begin{tabular}{|c|l|l|}
\hline & Nominal Controller & Extended Controller \\
\hline Gain margin & $6 d B$ & $7.9 d B$ \\
Phase margin & $90 \mathrm{deg}$ & $99.8 \mathrm{deg}$ \\
\hline
\end{tabular}

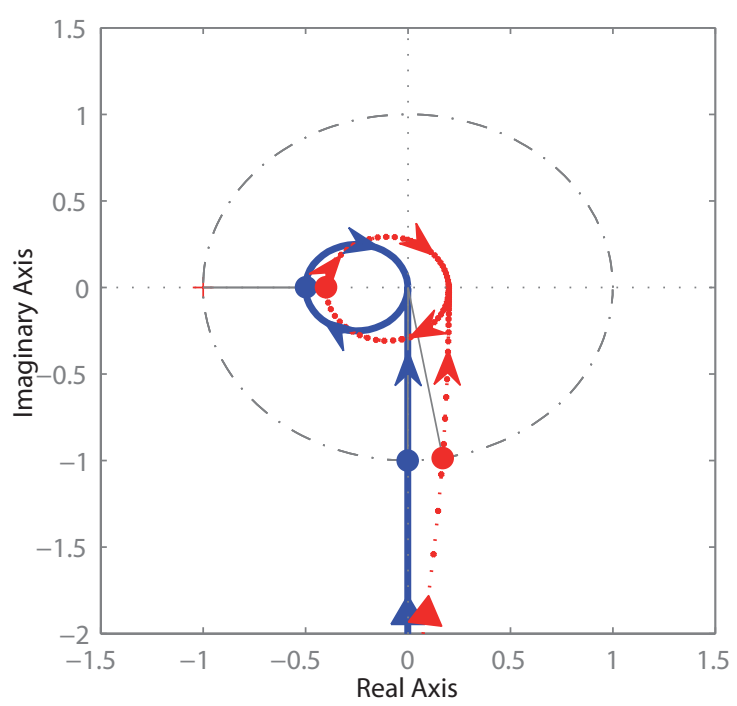

Fig. 3: Nyquist plot of the system given in (1) for the case of the original $\mathcal{L}_{1}$ controller (solide blue line) and the PID extended one (dotted red line).

\section{Effects of the PID gains on the stability}

The extended $\mathcal{L}_{1}$ controller was proven to satify the Nyquist criterion of stability for specific gains of the PID. We prove in this section that a wide variety of these gains can be selected while preserving the closed-loop stability. Going from the parameters used in the previous section, each gain of the PID is varied alone and the stability margins are computed for 


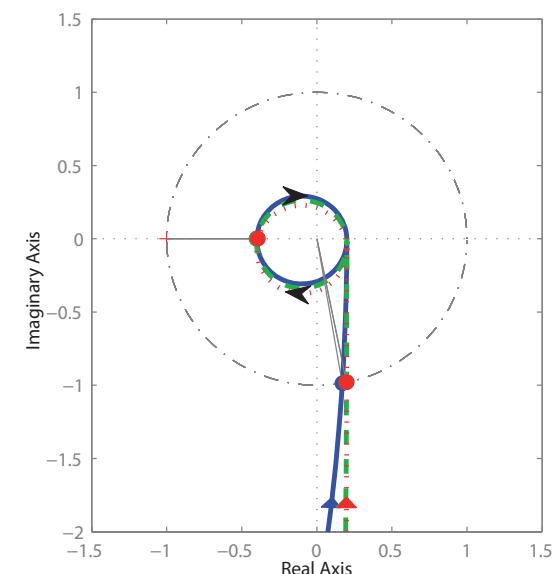

(a) Changing the proportional gain: $K_{p}=3$ in solid blue line, $K_{p}=15$ in dashed green line and $K_{p}=30$ in red dotted line.

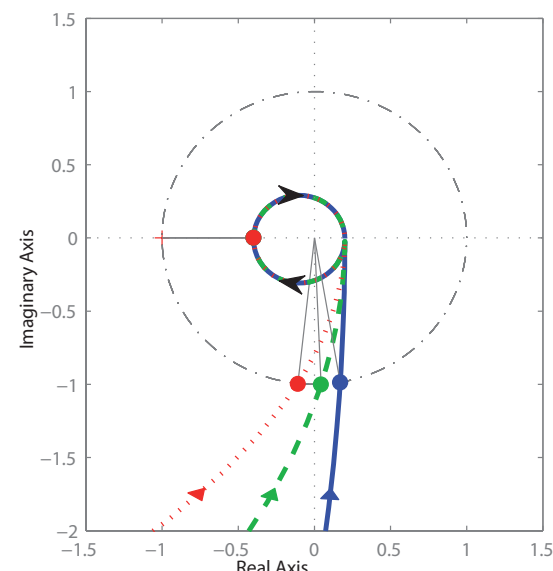

(b) Changing the integral gain: $K_{I}=0.5$ in solid blue line, $K_{I}=2.5$ in dashed green line and $K_{D}=5$ in red dotted line.

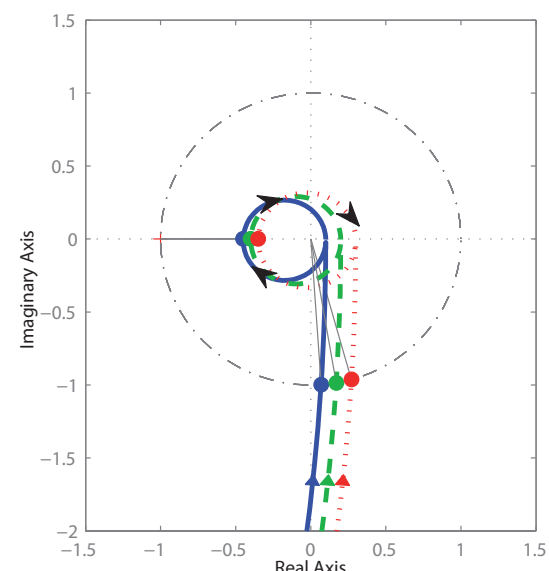

(c) Changing the proportional gain: $K_{D}=0.1$ in solid blue line, $K_{D}=0.2$ in dashed green line and $K_{D}=0.3$ in red dotted line.

Fig. 4: Nyquist plots of the open-loop system when changing a) the proportional gain, b) the integral gain and c) the derivative gain.

each case (cf. Table II, III,IV). In addition, the corresponding Nyquist plots are also drawn (cf. Fig. 4). We notice that augmenting the proportional gain has a very small effect on the stability margins given that the values in Table II nearly remain constant even after multiplying the proportional gain by 10 . The integral gain does not affect the gain margin (GM) but it lowers the phase margin (PM), whereas the derivative gain (cf. Table III) has a big impact on the values of the stability margins since a small increase in this parameter value increases both phase and gain margins. In conclusion, only the integral coefficient is prone to affect significantly the stability of the system and therefore should be chosen carefully. However, for the range of the above tested values, the phase and gain margins remain large enough to ensure the stability.

TABLE II: Values of the stability margins when the proportional gain is changed.

\begin{tabular}{|c|l||c|l||c|l|}
\hline Parameter & Value & Parameter & Value & Parameter & Value \\
\hline$K_{P}$ & 3 & $K_{P}$ & 15 & $K_{P}$ & 30 \\
$K_{I}$ & 0.5 & $K_{I}$ & 0.5 & $K_{I}$ & 0.5 \\
$K_{D}$ & 0.2 & $K_{D}$ & 0.2 & $K_{D}$ & 0.2 \\
$\mathrm{PM}$ & $100 \mathrm{deg}$ & $\mathrm{PM}$ & $101 \mathrm{deg}$ & $\mathrm{PM}$ & $101 \mathrm{deg}$ \\
$\mathrm{GM}$ & $7.9 d B$ & $\mathrm{GM}$ & $8 d B$ & $\mathrm{GM}$ & $8.1 d B$ \\
\hline
\end{tabular}

\section{Effects of the adaptation gain on the stability}

The adaptation gain was also varied to see its effect when an extended controller is used for the following chosen PID gains: $K_{P}=15, K_{I}=2.5$, and $K_{D}=0.2$. We notice from the Nyquist plot in Fig 5 and the Table $\mathrm{V}$ that the phase margins remained unchanged while we observe a slight decrease in
TABLE III: Values of the stability margins when the integral gain is changed.

\begin{tabular}{|c|l||c|l||c|l|}
\hline Parameter & Value & Parameter & Value & Parameter & Value \\
\hline$K_{P}$ & 3 & $K_{P}$ & 3 & $K_{P}$ & 3 \\
$K_{I}$ & 0.5 & $K_{I}$ & 2.5 & $K_{I}$ & 5 \\
$K_{D}$ & 0.2 & $K_{D}$ & 0.2 & $K_{D}$ & 0.2 \\
$\mathrm{PM}$ & $100 \mathrm{deg}$ & $\mathrm{PM}$ & $92 \mathrm{deg}$ & $\mathrm{PM}$ & $84 \mathrm{deg}$ \\
$\mathrm{GM}$ & $7.9 d B$ & $\mathrm{GM}$ & $7.9 d B$ & $\mathrm{GM}$ & $7.9 d B$ \\
\hline
\end{tabular}

TABLE IV: Values of the stability margins when the derivative gain is changed.

\begin{tabular}{|c|l||c|l||c|l|}
\hline Parameter & Value & Parameter & Value & Parameter & Value \\
\hline$K_{P}$ & 3 & $K_{P}$ & 3 & $K_{P}$ & 3 \\
$K_{I}$ & 0.5 & $K_{I}$ & 0.5 & $K_{I}$ & 0.5 \\
$K_{D}$ & 0.1 & $K_{D}$ & 0.2 & $K_{D}$ & 0.3 \\
$\mathrm{PM}$ & $94 \mathrm{deg}$ & $\mathrm{PM}$ & $100 \mathrm{deg}$ & $\mathrm{PM}$ & $106 \mathrm{deg}$ \\
$\mathrm{GM}$ & $6.9 d B$ & $\mathrm{GM}$ & $7.9 d B$ & $\mathrm{GM}$ & $9.1 d B$ \\
\hline
\end{tabular}

the gain margin which indicates that similarly to the original controller the adaptation gain does not alter the stability of the system for the extended $\mathcal{L}_{1}$.

TABLE V: Values of the stability margins when the adaptation gain is changed.

\begin{tabular}{|l|l|l|}
\hline Adaptation Gain & Phase Margin & Gain Margin \\
\hline 5000 & $8.9 \mathrm{~dB}$ & $101 \mathrm{deg}$ \\
10000 & $8.4 \mathrm{~dB}$ & $101 \mathrm{deg}$ \\
100000 & $8 \mathrm{~dB}$ & $101 \mathrm{deg}$ \\
\hline
\end{tabular}




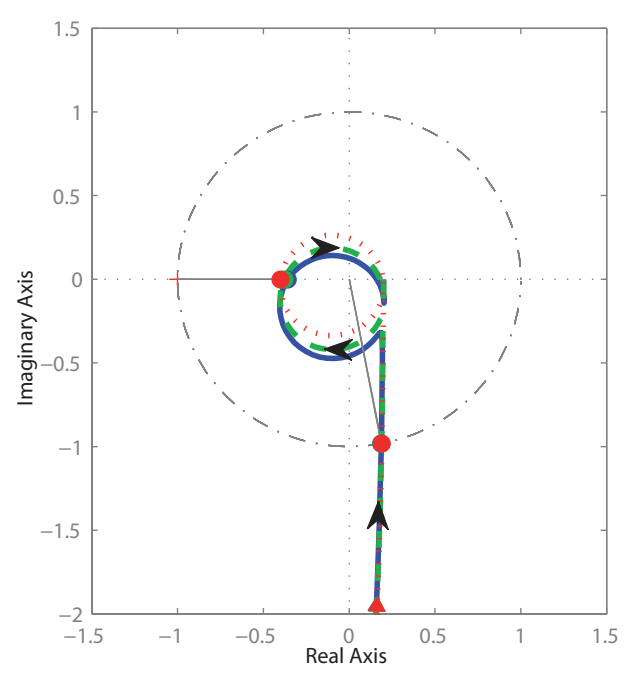

Fig. 5: Nyquist plot of the system given in (1) when changing the adaptation gain : $\Gamma=5000$ solid blue line, $\Gamma=10000$, dashed green line and $\Gamma=100000$ dotted red line.

\section{APPLICATION FOR DEPTH CONTROL OF AN UNDERWATER VEHICLE}

\section{A. Experimental setup}

The AC-ROV submarine (cf. Fig. 6) is an underactuated underwater vehicle. The propulsion system consists of six thrusters driven by DC motors controlling five degrees of freedom. Four horizontal thrusters control simultaneously translations along $x$ and $y$ axes and rotation around the $z$ axis (yaw angle). The two horizontal thrusters denoted 'Thruster 1' and 'Thruster 2' on Fig. 6 control depth position and pitch angle. The roll angle is unactuated but remains naturally stable due to the relative position of buoyancy and gravity centers. The robot weighs $3 \mathrm{~kg}$ and has a rectangular shape with height $203 \mathrm{~mm}$, length $152 \mathrm{~mm}$ and width $146 \mathrm{~mm}$. It has been modified by the LIRMM to become computer controllable. The different hardware components of the modified vehicle's hardware are detailed in [10].

The experiments have been performed in a $5 \mathrm{~m}^{3}$ pool. The tether has been sufficiently deployed to avoid inducing disturbances on the dynamics of the vehicle. The experiments were performed for depth control, where the position is measured by a depth sensor whereas the velocity of $z$ direction is estimated by an Alpha-Beta observer [11].

\section{B. Dynamic modeling of the system}

Using the SNAME notation and the representation proposed in [12], the depth dynamics of an underwater vehicle, expressed in the body-frame, is given by:

$$
\begin{gathered}
\dot{z}=J w \\
M_{z} \dot{w}+D_{z} w-\cos (\varphi) \cos (\vartheta)(W-B)=\tau_{z}+w_{d_{z}}
\end{gathered}
$$

where $\dot{z}$ and $w$ are the depth velocities in the earth-fixed frame and the body-fixed frame respectively. Due to the coupling in the dynamics, the Euler angles needed for

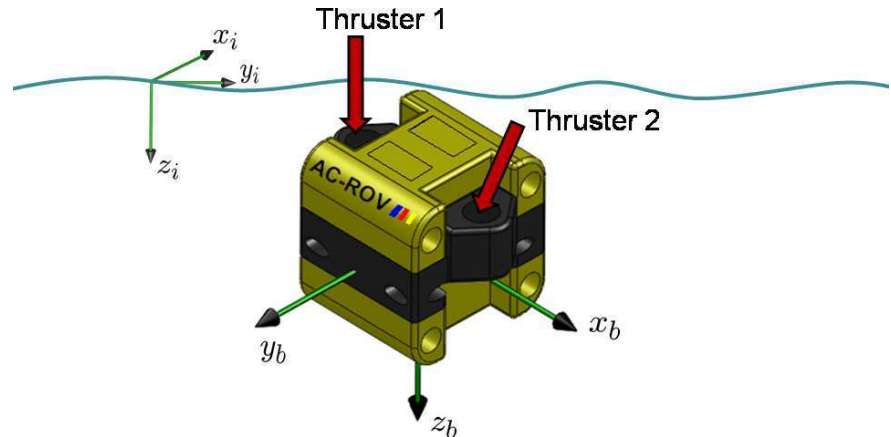

Fig. 6: View of the AC-ROV with the reference frames $\left(x_{i} y_{i} z_{i}\right.$ : earth-fixed frame, $x_{b} y_{b} z_{b}$ : body-fixed frame).

the studied dynamics are the roll $(\varphi)$ and the pitch $(\vartheta)$ expressed in the earth-fixed frame. $J(\varphi, \vartheta)=\cos (\varphi) \cos (\vartheta)$ is the transformation mapping from the body-fixed frame to the earth-fixed one. $M_{z}$ and $D_{z}$ denote the inertia (including added mass) and damping respectively. $W$ is the weight and $B$ the buoyancy. $w_{d_{z}}$ is the term representing the external disturbances and $\tau_{z}$ is the control input expressed in Newton and given by:

$$
\tau_{z}=T K u
$$

where $u \in \mathbb{R}^{2}$ is the vector of control inputs in volts (two thrusters are acting on the degree of freedom of interest, i.e depth), $K$ is the force coefficient in Newton. Volt ${ }^{-1}$ that has been experimentally identified. $T \in \mathbb{R}^{1 \times 2}$ is the actuators configuration matrix taking into account the position and orientation of the propellers, thus allowing to determine the associated forces in the body-fixed frame. Since the chosen reference frame is the earth one, the studied dynamics is then deduced from (7) and transformed into the following:

$$
M_{z}^{*}(\eta) \ddot{\eta}+D_{z}^{*}(v, \eta) \dot{\eta}+g_{z}^{*}(\eta)=\tau_{z}^{*}+w_{z}^{*} d
$$

The starred terms represent the model terms transformed from the body into the earth frame.

\section{Implementation of the extended $\mathcal{L}_{1}$ adaptive controller}

The extended $\mathcal{L}_{1}$ adaptive control architecture is applied to the depth $z$ in order to evaluate its closed-loop performance in terms of trajectory tracking and ability to reject external disturbances. Rewriting equation (9) in the state space form, we get:

$$
\left[\begin{array}{c}
\dot{\eta}_{1} \\
\dot{\eta}_{2}
\end{array}\right]=\left[\begin{array}{cc}
0 & 1 \\
0 & \frac{-D_{z}^{*}}{M_{z}^{*}}
\end{array}\right]\left[\begin{array}{l}
\eta_{1} \\
\eta_{2}
\end{array}\right]-\left[\begin{array}{c}
0 \\
\frac{g_{z}^{*}}{M_{z}^{*}}-\frac{w_{d_{z}}^{*}}{M_{z}^{*}}
\end{array}\right]+\left[\begin{array}{c}
0 \\
\frac{1}{M_{z}^{*}}
\end{array}\right] \tau_{z}^{*}
$$

with $\eta_{1}=z$ and $\eta_{2}=\dot{z}$. Rewriting (13) under the formalism of the $\mathcal{L}_{1}$ architecture as explained in [2] for the studied dynamics, we get:

$$
\begin{gathered}
{\left[\begin{array}{c}
\dot{\eta}_{1} \\
\dot{\eta}_{2}
\end{array}\right]=A_{m}\left[\begin{array}{l}
\eta_{1} \\
\eta_{2}
\end{array}\right]+\left[\begin{array}{c}
0 \\
\frac{1}{M_{z}^{*}}
\end{array}\right]\left(u_{a}+\theta(t)\|\eta(t)\|_{\mathcal{L}_{\infty}}+\sigma(t)\right)} \\
y=c\left[\begin{array}{l}
\eta_{1} \\
\eta_{2}
\end{array}\right]
\end{gathered}
$$




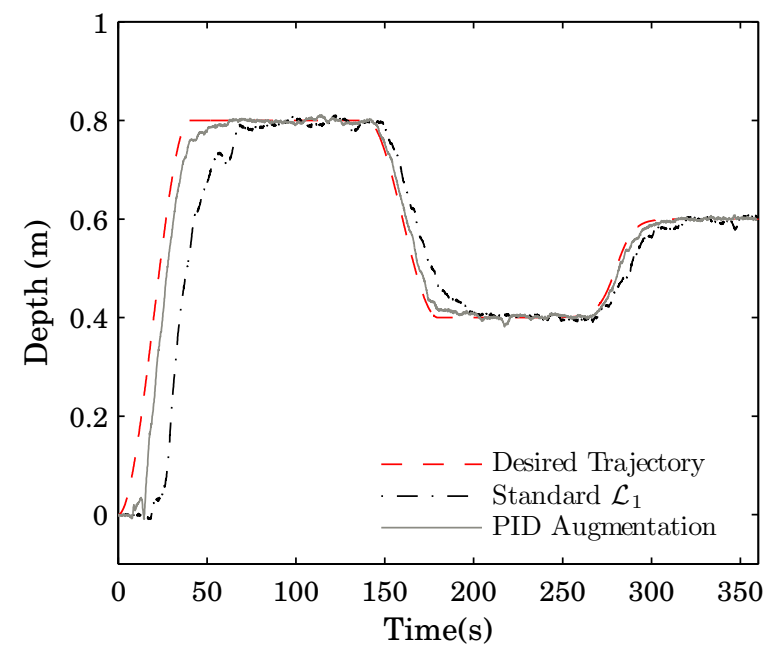

Fig. 7: Time history of the measured depth position $z$ of both controllers (standard and augmented $\mathcal{L}_{1}$ ), as well as the desired trajectory.

where $A_{m}=A-B_{m} k_{m} \in \mathbb{R}^{2 \times 2}$ is obtained from a choice of $k_{m}$ enabling the state matrix to be Hurwitz, $B_{m}=\left[0, \frac{1}{M_{z}^{*}}\right]^{T} \in$ $\mathbb{R}^{2 \times 1}$ and $c=\left[\begin{array}{ll}1 & 0\end{array}\right]$. The parameter $\theta \in \mathbb{R}$ represents the uncertainties on the damping coefficient and is given by: $\theta=\Delta\left(-D_{z}^{*}\right)$. The parameter $\sigma \in \mathbb{R}$ is a lumped parameter regrouping the gravitational and buoyancy forces as well as the external disturbances $\sigma=-g_{z}^{*}+w_{d_{z}}^{*}$. The expression $\|\eta(t)\|_{\mathcal{L}_{\infty}}$ refers to the infinity norm of the state vector at time $t$. The output is the depth $z$ and the control input is computed in the earth-fixed frame and should be transformed into the body fixed-frame such that:

$$
u=K^{-1} T^{-1} J^{T}\left(u_{a}+u_{m}+u_{P I D}\right) \in \mathbb{R}^{2}
$$

with $u_{a}(s)=-C(s)\left(\eta_{l}(s)-k_{g} r(s)\right)$, where $\eta_{l}=\hat{\theta}(t)\|\eta(t)\|_{\infty}+$ $\hat{\sigma}(t), k_{g}=\frac{-1}{c A_{m}^{-1} B_{m}}$ and $r(t)$ is the desired trajectory. $u_{m}(t)=$ $-k_{m}^{T} \eta(t)$ and $u_{P I D}$ as explained before.
Remark 1: Given that $A_{m}$ and $B_{m}$ are constant matrices, $M_{z}^{*}$ and $D_{z}^{*}$ in $A_{2}$ and $B_{2}$ were replaced by $M_{z}$ and $D_{z}$. This will guarantee for $A_{m}$ a constant desired dynamics. All the uncertainties will be compensated by the controlled parameters $\hat{\theta}$ and $\hat{\sigma}$ that are to be adapted.

\section{REAL-TIME EXPERIMENTAL RESULTS}

\section{A. Tracking error reduction}

Comparative experimental results between the original and the extended controller using a PID augmentation were performed and displayed in Fig. 7 in order to highlight the advantages the extended controller brings in terms of trajectory following. The robot is expected to follow a trajectory in $z$ going from the surface and reaching $0.8 \mathrm{~m}$ in 40 seconds. After remaining stable at this position for 100 seconds, the vehicle moves upwards to $0.4 \mathrm{~m}$ in 40 seconds and remains there for 70 seconds before going down to $0.6 \mathrm{~m}$ in 60 seconds. The original $\mathcal{L}_{1}$ adaptive controller needs around 65 seconds to reach the steady state depth (5\% of the final value) with no significant overshoot. The extended $\mathcal{L}_{1}$ adaptive controller reveals to be significantly faster with a convergence in 45 seconds and with no overshoot either. After the convergence to $0.8 \mathrm{~cm}$, a clear time lag is observed when the trajectory was changing with the $\mathcal{L}_{1}$ controller, while the augmented one is almost able to perfectly track the desired trajectory. We conclude from this experiment that the extended $\mathcal{L}_{1}$ adaptive controller is able to ensure a faster convergence and no overshoot is observed in the output tracking.

\section{B. Disturbance Rejection}

Experimental results were performed to validate the ability of the extended $\mathcal{L}_{1}$ controller to reject disturbances. The PID gains used in this experiment are $K_{p}=300, K_{I}=150$ and $K_{d}=250$ with an adaptation gain $\Gamma=10000$. Figure 8-(a) displays the evolution of the vehicle's position for a varying trajectory in presence of numerous perturbations occuring randomly along the trajectory. The robot is expected to follow the same trajectory as the one described in section

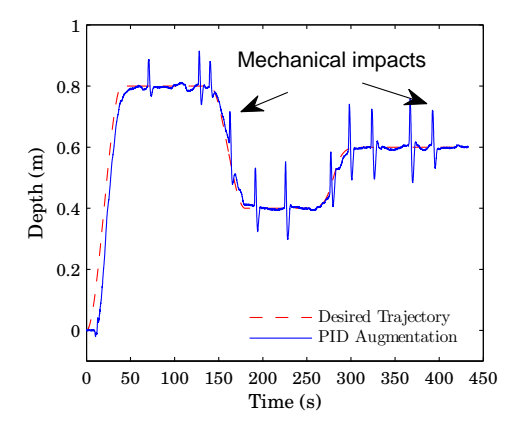

(a) Time history of the measured depth position $z$ of the extended $\mathcal{L}_{1}$ controller, as well as the desired trajectory.

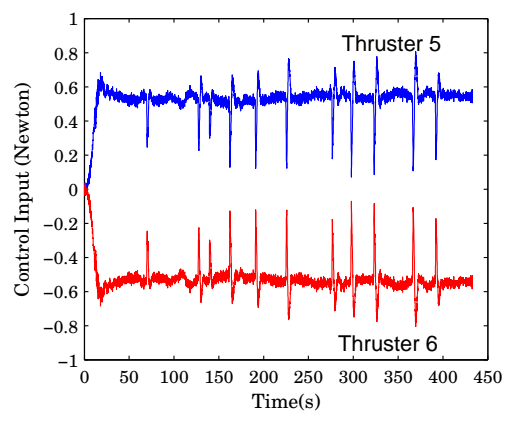

(b) Time history of the force exerted by the two thrusters controlling $z$

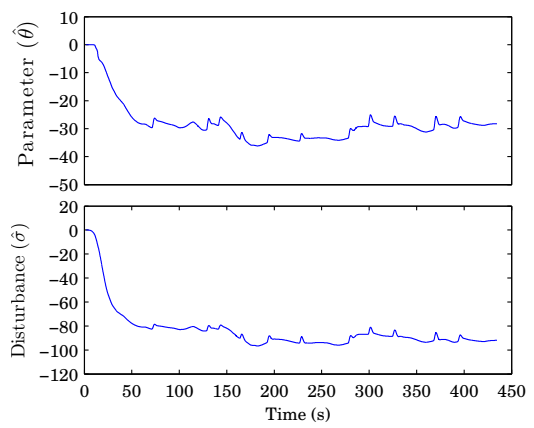

(c) Time history of the evolution of the parameters $\hat{\theta}$ and $\hat{\sigma}$

Fig. 8: Experimental results of the extended $\mathcal{L}_{1}$ controller in presence of disturbances: a) the depth response (b) the control input and (c) the parameters $\hat{\theta}$ and $\hat{\sigma}$. 
V.A. Eleven punctual disturbances (mechanical impacts) of different amplitudes were applied at various instants. The maximal one was of $16 \mathrm{~cm}$ applied at $t=367 \mathrm{~s}$ with a maxmial overshoot of $12 \mathrm{~cm}$. The recovery was of 8 seconds in average, i.e. the same performances than the ones observed with the original $\mathcal{L}_{1}$ controller in [10]. We can therefore deduce from this figure that along with the very goodt tracking of the varying trajectory, the rejection of external disturbances was ensured. The needed thrust was of $1.2 \mathrm{~N}$ in total $(0.6 \mathrm{~N}$ per thruster) (cf. Fig. 8-(b) and the peaks in this plot refer to the recovery procedure of the thruster in order to compensate the disturbance. Fig. 8-(c) displays the controlled parameters. Similarly to the original $\mathcal{L}_{1}$ controller there was a zero initialization for these parameters and therefore no necessity of having an a priori knowledge of the model. We also observe small peaks on the estimated values of the parameters at each imposed disturbance Fig. 8-(c). This effect is classical for $\mathcal{L}_{1}$ controllers and is due to the large adaptation gain $\Gamma$. This latter contributes to fast recovery in presence of disturbances.

\section{CONCLUSION}

In this paper, an extended version of the $\mathcal{L}_{1}$ adaptive controller is proposed. It is consituted of the original controller with a PID augmentation aiming on reducing the tracking error occuring when a varying trajectory is imposed on the system. The stability analysis is presented in order to prove the conservation of the stability margins of this new controller. Experimental results have been performed on an underwater vehicle for a varying trajectory in depth. Punctual disturbances have been added to the vehicle and the ability of their rejection has been demonstrated.

\section{REFERENCES}

[1] C.E Rohrs, L. Valavani, M. Athans, and G. Stein. Stability problems of adaptive control algorithms in the presence of unmodeled dynamics, 21st Conference on Decision and Control, Orlando,FL, December 1982.

[2] N. Hovakimyan and C. Cao. $\mathcal{L}_{1}$ Adaptive Control Theory, Society of Industrial and Applied Mathematics, 2010.

[3] E. Kharisov and N. Hovakimyan. Comparison of several adaptive controllers according to their robustness metrics, AIAA Guidance, Navigation and Control Conference, Toronto, Canada, August 2010.

[4] E. Xargay, N. Hovakimyan, and C. Cao. Benchmark problems of adaptive control revisited by $\mathcal{L}_{1}$ adaptive control. pages $31-36,17$ th Mediterranean Conference on Control and Automation, Thessaloniki, Grece, June 2009.

[5] J. Vanness, E. Kharisov, and N. Hovakimyan. $\mathcal{L}_{1}$ adaptive control with proportional adaptation law. pages 1919 - 1924, American Control Conference, Montreal, Canada, June 2012.

[6] E. Kharisov and N. Hovakimyan. Generalization of $\mathcal{L}_{1}$ adaptive control architecture for switching estimation laws pages 1907 - 1912, American Control Conference, Montreal, Canada, June 2012.

[7] V Natarajan and J Bentsman. Adaptive observers with projection operator and $\mathcal{L}_{1}$ adaptive controllers for infinite dimensional systems. pages 3002 - 3007, American Control Conference, Montreal, Canada, June 2012.

[8] M. Naghnaeian, P.G Voulgaris and N. Hovakimyan. On robustness of $\mathcal{L}_{1}$ adaptive control with time varying perturbations \& filter design. pages 1937 - 1942, American Control Conference, Montreal, Canada, June 2012.

[9] D. Maalouf, A. Chemori and V. Creuze, A new extension of the $\mathcal{L}_{1}$ adaptive controller to drastically reduce the tracking time lags Submitted to the 9th IFAC Symposium on Nonlinear Control Systems, NOLCOS'13, Toulouse, France, 2013.
[10] D. Maalouf, V. Creuze, and A. Chemori. A novel application of multivariable $\mathcal{L}_{1}$ adaptive control: From design to real-time implementation on an underwater vehicle. In IEEE/RSJ IROS'12, Algarve, Portugal, 2012.

[11] R. Penoyer. The alpha-beta filter. C User's Journal, 11(7):73-86, 1993.

[12] T.I. Fossen. Marine Control Systems: Guidance, Navigation and Control of Ships, Rigs and Underwater Vehicles. Marine Cybernetics, As,Trondheim, 2002. 